\author{
Nota Científica \\ (Short Communication)
}

\title{
REGISTRO DEL CIEMPIÉS SCOLOPENDRA POLYMORPHA WOOD, 1861 (CHILOPODA: SCOLOPENDROMORPHA: SCOLOPENDRIDAE) EN LA ISLA MARÍA CLEOFAS, OCÉANO PACÍFICO DE MÉXICO
}

\author{
RECORD OF CENTIPEDE SCOLOPENDRA POLYMORPHA WOOD, 1861 \\ (CHILOPODA: SCOLOPENDROMORPHA: SCOLOPENDRIDAE) \\ IN MARIA CLEOFAS ISLAND, PACIFIC OCEAN, MEXICO
}

\author{
Ubaldo Sebastián FLORES-GUERRERO, ISMael Eduardo HUERTA DE LA BARRERA \\ y Fabio Germán CUPUL-MAGAÑ *
}

\begin{abstract}
Centro Universitario de la Costa, Universidad de Guadalajara. Av. Universidad 203, Delegación Ixtapa, C.P. 48280, Puerto Vallarta, Jalisco, México < sebastian_toci@hotmail.com >, <atropofauna3000@gmail.com>, $<$ fabiocupul@gmail.com>

*Autor para correspondencia:<fabiocupul@gmail.com>
\end{abstract}

Recibido: 31/10/2017; aceptado: 01/12/2017; publicado en línea: 16/03/2018

Editor responsable: Pedro Reyes Castillo

Flores-Guerrero, U. S., Huerta de la Barrea, I. E. y Cupul Magaña, F. G. (2018) Registro del ciempiés Scolopendra polymorpha Wood, 1861 (Chilopoda: Scolopendromorpha: Scolopendridae) en la Isla María Cleofas, Océano Pacifico de México. Acta Zoológica Mexicana (n.s.), 34(1): 1-4.

RESUMEN. Durante una visita a la Isla María Cleofas, en el archipiélago de las Islas Marías, ubicado en la desembocadura del Golfo de California, México, se recolectaron tres especímenes del ciempiés tigre Scolopendra polymorpha Wood, 1861 (ejemplares de longitud: $73 \mathrm{~mm}, 91 \mathrm{~mm}$ y $125 \mathrm{~mm}$ ). Hasta donde sabemos, estos ejemplares representan el primer registro confirmado de la especie para la Isla María Cleofas. Probablemente, el ciempiés tigre y la fauna de ciempiés en general de la Isla María Cleofas provienen de áreas continentales adyacentes.

Scolopendra polymorpha Wood, 1861 es una de las 13 especies de ciempiés escolopendromorfos (Scolopendridae) que habitan en México (Shelley, 2006; Cupul-Magaña, 2013). Se distribuye al oeste del río Missouri y en la región suroeste de los Estados Unidos de América (Shelley, 2002), así como en los estados mexicanos de Baja California, Baja California Sur, Chihuahua, Coahuila, Ciudad de México, Durango, Guerrero, Hidalgo, Jalisco, Michoacán, Morelos, Nayarit (Islas Marías), Nuevo León, Oaxaca, Puebla, Querétaro, Sinaloa, Sonora, Tamaulipas
Flores-Guerrero, U.S., Huerta de la Barrea, I. E., \& Cupul Magaña, F. G. (2018) Record of centipede Scolopendra polymorpha Wood, 1861 (Chilopoda: Scolopendromorpha: Scolopendridae) in Maria Cleofas Island, Pacific Ocean, Mexico. Acta Zoológica Mexicana (n.s.), 34(1): 1-4.

ABSTRACT. During a visit to María Cleofas Island, in the Marías Islands archipelago, located in the mouth of the Gulf of California, Mexico, three specimens of the tiger centipede Scolopendra polymorpha Wood, 1861 were collected (exemplars length: $73 \mathrm{~mm}, 91 \mathrm{~mm}$, and $125 \mathrm{~mm}$ ). To our knowledge, these specimens represent the first confirmed record of the species from María Cleofas Island. Probably, the tiger centipede, and centipede fauna in general from María Cleofas Island, comes from continental adjacent areas.

y Zacatecas (Pocock, 1895-1910; Shelley, 2002; CupulMagaña, 2013; Fig. 1). También, se ha introducido en las islas Hawái, posiblemente se encuentre en Belice (Shelley, 2006) y con un registro reciente en Iraq (Ahmed \& Hussen, 2016).

Su talla máxima registrada es de $135 \mathrm{~mm}$ (Chamberlin, 1915); antenas con 21 a 31 segmentos, donde los primeros tres a 16 basales con escasas setas; coxoesternito forcipular sin sutura transversal; terguito 1 con sutura transversal y dos suturas paramedianas; terguitos 8 a 12 con márge- 


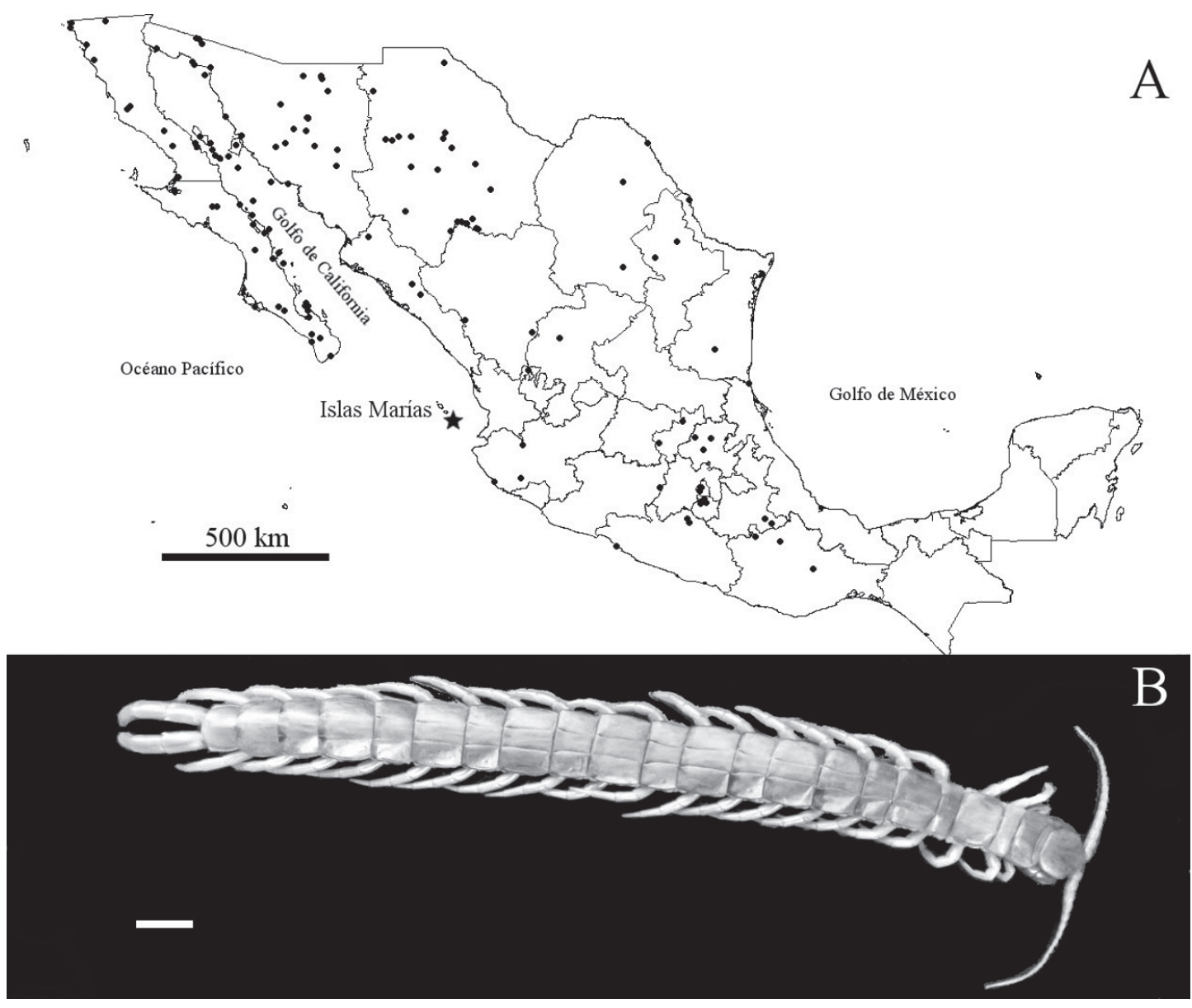

Figura 1. A) Registros conocidos de Scolopendra polymorpha en México (puntos negros = registros previos; estrella negra = primer registro en la Isla María Cleofas). B) Vista dorsal de ejemplar preservado de $125 \mathrm{~mm}$ de $S$. polymorpha de la Isla María Cleofas. Marca de escala $1 \mathrm{~cm}$.

nes laterales, poco frecuentes hasta los terguitos 16 a 17; terguito 21 con sutura mediodorsal; prefémures de las patas terminales con ocho a diez espinas ventrolaterales, ventromediales, mediales y siete a trece dorsomediales; procesos espinosos de los prefémures con cuatro espinas, aunque puede existir mucha variación, desde cero hasta doce espinas (Kraepelin, 1903; Chamberlin, 1912; Attems, 1930; Shelley, 2002).

Estudios específicos en las islas de México sobre la presencia de especies de ciempiés escolopendromorfos, y de miriápodos en general, son escasos. El primer trabajo fue el de Pocock (1895-1910), quien cita a S. polymorpha en las Islas Marías, pero sin especificar en cuál de ellas se descubrió. Chamberlin (1923), recolectó siete especies de quilópodos, con S. polymorpha como la más abundante, y cuatro de diplópodos para 19 islas del Golfo de California. En algunas otras islas del mismo Golfo, Cupul-Magaña y Bueno-Villegas (2007) encontraron al ciempiés Rhysida longipes en la Isabel; mientras que Cupul-Magaña (2010) registró a S. polymorpha en Partida Norte y San Lorenzo, así como a $S$. viridis en Ángel de la Guarda, San Marcos y Socorro. En las Marías, Cupul-Magaña (2015) documentó una especie de ciempiés en la María Madre y cuatro en la María Cleofas; en esta última, encontró dos especies de ciempiés del género Scolopendra: Scolopendra morsitans Linnaeus, 1758 y S. viridis Say, 1821.

En esta nota se registra por primera vez la presencia de $S$. polymorpha en la Isla María Cleofas, una de las cuatro islas que conforman la Reserva de la Biosfera del archipiélago de las Islas Marías en el Pacífico de México y que territorialmente pertenece al estado de Nayarit (CONABIO 2007). En María Cleofas (coordenada media $21^{\circ} 18^{\prime} 53.45^{\prime}$ " N y $106^{\circ} 14^{\prime} 59.86^{\prime \prime} \mathrm{O}$; superficie de $1,882.973493$ ha; $132 \mathrm{~km}$ desde San Blas, Nayarit), la vegetación representativa es el bosque tropical subcaducifolio (CONABIO, 2007). 
Los ejemplares se recolectaron a partir de búsquedas visuales diurnas y nocturnas entre el 22 y 25 de agosto de 2017. Su identificación se realizó con la consulta del trabajo de Shelley (2002) y su sexo no fue determinado. Posteriormente, fueron sacrificados y colocados en frascos con alcohol etílico al 70\% para su depósito en la Colección Entomológica del Centro de Estudios en Zoología de la Universidad de Guadalajara (CZUG) en Zapopan, Jalisco. Para elaborar el mapa de localidades de registro de S. polymorpha en México, se utilizó el Sistema de Información Biótica $\subset$ y la base de datos (no publicada) del Catálogo de Autoridades Taxonómicas (CAT) de Myriapoda en México.

Material examinado: México, Golfo de California, Isla María Cleofas; 22.VIII.2017; colector I. Huerta; talla 91 $\mathrm{mm}$; ancho del décimo terguito (adt) $6 \mathrm{~mm}$; antena derecha con 24 segmentos e izquierda con 25 , con los seis basales con escasas setas; terguitos 12 al 21 marginados; procesos coxopleurales de la coxopleura con siete espinas apicales y una lateral; prefémures de las patas terminales con 25 espinas, los procesos prefemorales apicales con dos espinas el derecho y tres el izquierdo. México, Golfo de California, Isla María Cleofas; 23.VIII.2017; colector I. Huerta; talla $73 \mathrm{~mm}$; adt de $5 \mathrm{~mm}$; antenas con 27 segmentos, con los cinco basales con escasas setas; terguitos 14 al 21 marginados; procesos coxopleurales de la coxopleura con siete espinas apicales y una lateral; prefémures de las patas terminales con 46 espinas el derecho y 38 espinas el izquierdo, los procesos prefemorales con tres espinas. México, Golfo de California, Isla María Cleofas; 25.VIII.2017; colector I. Huerta; talla $125 \mathrm{~mm}$; adt de $11 \mathrm{~mm}$; antena derecha con 23 segmentos e izquierda con 21 , con los cinco basales con escasas setas; terguitos 12 al 21 marginados; procesos coxopleurales del coxopleurón derecho con una espina apical e izquierdo con siete apicales y una lateral; prefémures de las patas terminales con 23 espinas el derecho y 21 espinas el izquierdo, los procesos prefemorales con cuatro espinas en el derecho y tres en el izquierdo.

En la mayoría de los estados mexicanos que bordean al Golfo de California (Fig. 1), se ha registrado a S. polymorpha; por lo que es posible establecer que los individuos que colonizan actualmente la isla tengan un origen continental. Lo anterior se apoya en el hecho de que, geológicamente, el archipiélago es de origen continental y al separase durante la apertura de la boca del Golfo de California en el Plioceno, hace aproximadamente cinco millones de años (Carreño \& Helenes, 2002), tal vez favoreció el aislamiento insular de los ciempiés. Evidencias sobre las áreas continentales adyacentes como fuente de diversidad biológica de las islas del Golfo, en especial de artrópodos, se han observado para la fauna de hormigas y de coleópteros tenebriónidos (Boulton \& Ward, 2002; Sánchez-Piñero \& Aalbu, 2002). También, existe la posibilidad de que en el pasado un ciempiés se dispersara desde el continente hacia la isla al viajar, por ejemplo, dentro de la grieta de un coco que se revuelca en una playa, y sobrevivir la travesía oceánica (Thornton, 2007).

En el archipiélago se han registrado especies endémicas de plantas y artrópodos, así como subespecies de aves, mamíferos y reptiles (Martínez et al., 2010); por lo que cabría la posibilidad de encontrar esto mismo para $S$. polymorpha $\mathrm{u}$ otras especies de ciempiés dado su aislamiento. Sin embargo, aunque se observó variabilidad en algunos caracteres morfológicos de los ejemplares de las Isla María Cleofas, como marginación de los terguitos, espinas del coxopleurón o de los prefémures del último par de patas; se requerirá de la revisión de un mayor número de especímenes insulares, su comparación con ejemplares continentales (tanto de la península como del continente) y estudios moleculares para llegar a alguna conclusión al respecto.

La recolecta de un gran ejemplar de $125 \mathrm{~mm}$ de longitud, supera el registro previo conocido para las Islas Marías de 85 mm (Pocock, 1895-1910). Además, esta medición se aproxima a la talla máxima observada para $S$. polymorpha de $135 \mathrm{~mm}$. Por otra parte, es probable que la especie colonizara la Isla María Cleofas porque en ella dispone de los recursos alimenticios y de condiciones ambientales favorables (o esté aclimatada), como humedad, temperatura y vegetación de calidad (Voigtländer, 2011), para desarrollarse y sobrevivir. Asimismo, con el hallazgo del ciempiés tigre, se eleva a seis el número de especies conocidas de quilopodofauna para esta área insular.

AGRADECIMIENTOS. A la Comisión Nacional de Seguridad (CNS), Secretaria de Medio Ambiente y Recursos Naturales (SEMARNAT), Secretaria de Marina (SEMAR), Complejo penitenciario federal Islas Marías y a la Asociación Civil Protección y Restauración de Islas y Zonas Naturales (PROZONA, AC.), por su apoyo para la realización de este trabajo. A la Comisión Nacional para el Conocimiento y Uso de la Biodiversidad (CONABIO) por permitirnos el uso de la base de datos del catálogo CAT de Myriapoda en México. A los revisores anónimos por sus comentarios.

\section{LITERATURA CITADA}

Ahmed, S. T. \& Hussen, F. Sh. (2016) First record of two Scolopendromorph centipedes; Scolopendra subspinipes Leach, (1815) and 
Scolopendra polymorpha Wood, (1861) with additional notes on Scolopendra cingulata Latreille, (1829) in Iraq. ZANCO Journal of Pure and Applied Sciences, 82(6), 471-428.

Attems, C. (1930) Myriapoda 2. Scolopendromorpha. Das Tierreich, $54,1-308$.

Boulton, A. M. \& Ward, R. F. (2002) Tenebrionid beetles. Pp. 129153. En: T.J. Case, M.L. Cody \& E. Ezcurra (Eds.). A new island biogeography of the Sea of Cortés. Oxford University Press, Oxford.

Carreño, A. L. \& Helenes, J. (2002) Geology and ages of the islands. Pp. 14-40. En: T.J. Case, M.L. Cody \& E. Ezcurra (Eds.). A new island biogeography of the Sea of Cortés. Oxford University Press, Oxford.

Chamberlin R. V. (1912) New North American chilopods and diplopods. Annals of the Entomological Society of America, 5, 141172 .

Chamberlin, R. V. (1915) New chilopods from Mexico and the West Indies. Bulletin of the Museum of Comparative Zoology at Harvard College, 59(8), 495-541.

Chamberlin, R. V. (1923) On chilopods and diplopods from islands in the Gulf of California. Proceedings of the California Academy of Sciences, 12(18), 389-407.

CONABIO. (2007). Programa de conservación y manejo Reserva de la Biosfera Islas Marías, México. SEMARNAT-CONANP, México.

Cupul-Magaña. F. G. (2010) An annotated list of the centipedes (Chilopoda) in the National Collection of Arachnids, Instituto de Biología, Universidad Nacional Autónoma de México. Insecta Mundi, 0125, 1-10.

Cupul-Magaña, F. G. (2013). La diversidad de los ciempiés (Chilopoda) de México. Dugesiana, 20(1): 17 -41.

Cupul-Magaña, F. G. (2015) Ciempiés (Myriapoda: Chilopoda) de las islas María Madre y María Cleofas, Archipiélago de las Islas Marías, Pacífico de México. Boletín de la Sociedad Entomológica Aragonesa (S.E.A.), 56, 335-338.

Cupul-Magaña, F. G. \& Bueno-Villegas, J. (2007) Primer registro de Rhysida longipes (Chilopoda: Scolopendromorpha: Scolopendridae) en el Parque Nacional Isla Isabel, Golfo de California, México. Dugesiana, 14(1), 39-41.

Kraepelin, K. (1903) Revision der Scolopendriden. Mitteilungen aus dem Naturhistorischen Museum in Hamburg, 20, 1-276.

Martínez, J., Mason-Romo, E. \& Ceballos, G. (2010) Islas Marías. Pp. 433-440. En: G. Ceballos, L. Martínez, A. García, E. Espinoza, J. Bezaury Creel \& R. Dirzo (Eds.). Diversidad, amenazas y áreas prioritarias para la conservación de las selvas secas del Pacifico de México. CONABIO y Fondo de Cultura Económica, México.

Pocock, R. I. (1895-1910) Chilopoda and Diplopoda, volume 14. Pp. 1-217. En: F. D. Godman \& O. Salvin (Eds.). Biologia CentraliAmericana. Taylor and Francis, London.

Sánchez-Piñero, F. \& Aalbu, P.S. (2002) Ants. Pp. 112-128. En: T.J. Case, M.L. Cody \& E. Ezcurra (Eds.). A new island biogeography of the Sea of Cortés. Oxford University Press, Oxford.

Shelley, R. M. (2002) A synopsis of the North American centipedes of the Order Scolopendromorpha (Chilopoda). Virginia Museum of Natural History Memoir, 5, 1-108.

Shelley, R. M. (2006) A chronological catalog of the New World species of Scolopendra L., 1758 (Chilopoda: Scolopendromorpha: Scolopendridae). Zootaxa, 1253, 1-50.

Thornton, I. (2007) Island colonization: The origin and development of island communities. Cambridge University Press, Cambridge.

Voigtländer, K. (2011) Chilopoda-Ecology. Pp. 309-325. En: A. Minelli (Ed.). The Myriapoda: Treatise on zoology - anatomy, taxonomy, biology. Volume 1. Brill, Leiden. 